\title{
Registros sobre dor pós-operatória em crianças: uma análise retrospectiva de hospitais de Londrina, PR, Brasil*
}

\author{
Notes of post-operative pain in children: a retrospective analyses of hospitals from Londrina, PR, Brazil
}

Registros sobre el dolor post operatorio en niños: un análisis retrospectivo en hospitales de Londrina, PR, Brasil

\author{
Mauren Teresa Grubisich Mendes Tacla', \\ Miyeko Hayashida", Regina Aparecida Garcia de Lima" \\ 'Universidade Estadual de Londirna, Londrina, PR \\ "Universidade de São Paulo, Escola de Enfermagem de Ribeirão Preto, Ribeirão Preto, SP
}

Submissão: 02/02/2007

Aprovação: 10/01/2008

\section{RESUMO}

Toda cirurgia implica em lesão de tecidos, manipulação de estruturas e órgãos, sendo a dor uma conseQüência desse processo, Que pode ser minimizada. Este estudo tem como objetivo caracterizar, retrospectivamente, o processo de manejo da dor pós-operatória a partir dos registros contidos em 300 prontuários de 280 crianças de 0 a 14 anos submetidas à cirurgia no ano de 2004 em três hospitais de Londrina, PR. O estudo indicou a existência de poucos registros sobre dor pós-operatória realizados por enfermeiras, sendo a maioria realizada por auxiliares ou técnicos de enfermagem. Esses achados evidenciam a necessidade de realização de pesquisas sobre manejo da dor pediátrica e os fatores Que o influenciam.

Descritores: Dor pós-operatória; Criança; Enfermagem pediátrica.

\section{ABSTRACT}

All surgical procedures involve tissue lesions, manipulation of structures and organs, causing pain which can be minimized during the process. The objective of this study is to characterize, retrospectively, the process of managing post-operative pain based on 300 medical records of 280 childre, who underwent surgery in 2004, in 3 hospitals located in Londrina, PR, Brazil. The study revealed that only a few nurses document post-operative pain, and that the majority of the records were made by nursing aides or technicians. The results have shown the need for research on pediatric pain management and the factors affecting them.

Descriptors: Pain; post-operative; Child; Pediatric nursing.

\section{RESUMEN}

Toda cirugía implica en lesión de tejidos, manipulación de estructuras y órganos, siendo el dolor una consecuencia de ese proceso, Que puede ser minimizada. El presente estudio tiene como objetivo caracterizar, retrospectivamente, el proceso del manejo del dolor pos operatorio a partir de los registros contenidos en 300 prontuarios de 280 niños de 0 a 14 años sometidos a cirugía el año 2004 en tres hospitales de Londrina, PR, Brasil. El estudio indicó la existencia de pocos registros realizado por enfermeras, y la mayoría fue realizada por auxiliares o técnicos de enfermería. Los resultados evidencian la necesidad de la realización de pesquisas sobre el manejo del dolor pediátrico y los factores que lo influencian.

Descriptores: Dolor post operatorio; Niño; Enfermería pediátrica.

"Trabalho extraído da tese de doutorado intitulada "Cuidado à criança com dor pós-operatória: experiências de enfermeiras pediatras", apresentada à Escola de Enfermagem de Ribeirão Preto da Universidade de São Paulo junto ao Programa de Pós-Graduação Enfermagem em Saúde Pública.

Correspondência: Mauren Teresa Grubisich Mendes Tacla. Universidade Estadual de Londrina, Departamento de Enfermagem. Av. Robert Kock, 60. Vila Operária. CEP 86038-440 - Londrina, PR. 


\section{INTRODUÇÃO}

Atualmente, muito se sabe sobre o manejo seguro e efetivo da dor em crianças. Esse conhecimento, no entanto, é pouco empregado na prática clínica diária. PesQuisas ao redor do mundo foram responsáveis por inúmeras descobertas em relação a causas, mecanismos e tratamento da dor ${ }^{(1-3)}$.

Ainda são comuns, no entanto, crenças de caráter negativo sobre a dor em crianças ${ }^{(4-7)}$ sendo as mais comuns: a criança não sente dor ou sente menos que o adulto; medo de Que as crianças se viciem em opióides; medo da depressão respiratória; o comportamento de dor da criança pode ser controlado pela contenção; as crianças toleram a dor melhor Que os adultos; as crianças acostumam-se à dor ou a procedimentos dolorosos; manifestações comportamentais refletem a intensidade da dor; as crianças não conseguem dizer onde está a dor; é difícil e toma muito tempo avaliar a dor da criança e a dor é importante no desenvolvimento, por exemplo, constrói o caráter.

Tais crenças estão sendo colocadas em dúvida em função dos avanços da fisiologia e da fisiopatologia da dor. O fenômeno da equivalência dolorosa entre o adulto e a criança não existe, o que não anula o fato de Que a criança tenha dor. As vias neurofisiológicas de nocicepção para o córtex já estão desenvolvidas, mesmo nos prematuros ${ }^{(8)}$. Não é verdadeira a afirmação de Que a criança não tem uma resposta, nem se lembra das experiências de dor com o mesmo grau Que o adulto, visto Que muitos trabalhos de revisão sobre a utilização de analgésicos não-opióides e opióides têm afastado muitas dessas crenças, possibilitando o tratamento da dor em pediatria de maneira mais fácil e efetiva ${ }^{(9)}$.

Outros fatores que contribuem para o alívio inadequado da dor em pediatria são: a utilização de doses sub-terapêuticas de analgésicos e sedativos; a prescrição de analgésicos apenas conforme o necessário e não em horários regulares; os intervalos demasiadamente longos entre as doses e a utilização de placebos ${ }^{(6)}$.

No caso da dor pós-operatória, deve-se considerar Que toda cirurgia implica em lesão de tecidos, manipulação de estruturas e órgãos, sendo a dor uma conseQüência desse processo, que pode, porém, ser minimizada. O período pós-operatório (PO), tão comum numa unidade pediátrica, pode ser vivenciado pela criança de forma menos traumática se os profissionais responsáveis pelo seu cuidado estiverem sensibilizados para a importância da avaliação e alívio da dor e conhecerem estratégias adeQuadas para o seu tratamento.

Muitas das crianças submetidas a cirurgias ainda não recebem tratamento adequado para o alívio da dor pós-operatória, apesar do fato de Que a analgesia poderia ser efetuada com relativa facilidade e de forma segura, não havendo, portanto, razões para o nãotratamento dos Quadros álgicos pós-operatórios ${ }^{(10)}$.

A dor pós-operatória tem causa identificável, etiologia bem definida e, em decorrência disso, por ser uma dor previsível, esperase Que seja autolimitada, devendo seguramente diminuir ou cessar em pouco tempo. Ao longo do desenvolvimento da cirurgia, a dor pós-operatória foi Quase ignorada, sendo universalmente reconhecido o fato de Que ela recebe subtratamento Que pode ser, em grande parte, atribuído ao temor do emprego de drogas opiáceas $^{(2,11)}$.

Outras razões para seu tratamento inadequado são: crença de Que a dor é inevitável após toda intervenção cirúrgica; desconhecimento dos benefícios fisiológicos da analgesia pósoperatória; temor na utilização de drogas mais potentes; receio de induzir depressão respiratória e dependência física ou psíquica do paciente com o uso de opiáceos ${ }^{(11,12)}$

O tratamento da dor pós-operatória, além das razões humanitárias, reduz reações secundárias induzidas pelo traumatismo, isto é, os reflexos somáticos e neurovegetativos Que podem influenciar negativamente a atividade de vários órgãos e contribuir no aumento da morbidade ${ }^{(13)}$. Vale destacar Que em QualQuer faixa etária a dor aumenta a morbidade e a mortalidade dos doentes, dificultando a recuperação e reparação de processos mórbidos clínicos ou cirúrgicos ${ }^{(10)}$.

Apesar dos significativos avanços nas áreas da fisiopatologia da dor, da farmacologia dos analgésicos e do desenvolvimento de novas técnicas para o controle da dor pós-operatória, muitos pacientes continuam recebendo tratamento inadequado. Pacientes Que recebem tratamento adeQuado da dor após a cirurgia encaram a experiência de forma mais otimista, motivo pelo Qual é importante criar e manter rotinas, visando dar o máximo conforto ao paciente, tratando de forma adequada sua dor ${ }^{(12)}$.

A avaliação da dor também inclui o registro das informações colhidas e das estratégias utilizadas para seu controle. Ao registrar os dados relacionados ao manejo da dor a equipe de enfermagem permite Que os mesmos sejam compartilhados entre os diversos turnos e a equipe multiprofissional, possibilitando uma melhor assistência. A comunicação entre o paciente e os profissionais Que o atendem também é importante para a compreensão do Quadro álgico e de seu alívio ${ }^{(14)}$. No caso das crianças, é indispensável a inclusão da família na obtenção de dados sobre a dor e a efetividade das ações Que buscam seu controle.

Tendo em vista as considerações apresentadas, este estudo teve como objetivo caracterizar, retrospectivamente, o processo de manejo da dor pós-operatória a partir dos registros contidos nos prontuários de crianças submetidas à cirurgia em três hospitais de uma cidade do interior do Paraná.

\section{MÉTODO}

Trata-se de um estudo retrospectivo em Que foram pesQuisados prontuários de crianças na faixa etária de 0 a 14 anos, submetidas a cirurgias em três hospitais de Londrina-PR, durante o ano de 2004, com permanência nas unidades pediátricas dos referidos hospitais por um período mínimo de 24 horas, contados a partir do horário de término da cirurgia. A escolha dos hospitais deveu-se ao fato de Que todos recebem crianças com indicação cirúrgica em suas unidades de internação pediátrica. Um deles é hospital-escola geral e público de grande porte, outro é hospital geral privado também de grande e o último trata-se de um hospital pediátrico privado de médio porte.

As crianças tinham necessariamente que sair do centro cirúrgico diretamente para a unidade de pediatria. Prontuários incompletos e de crianças Que sofreram procedimentos diagnósticos ou terapêuticos não cirúrgicos realizados sob anestesia no centro cirúrgico foram excluídos.

Os dados foram coletados no período de maio de 2005 a fevereiro de 2006, com a utilização de instrumento adaptado de

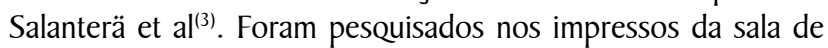


operação (SO), da sala de recuperação pós-anestésica (SRPA), nas prescrições médicas e de enfermagem dados sobre o manejo da dor, entre eles: prescrição médica de analgésicos e sedativos no pré, trans e pós-operatórios, registros de enfermagem sobre queixas de dor no local da cirurgia, avaliação da dor e as medidas adotadas para o seu alívio. A coleta dos dados foi feita a partir do registro pré-anestésico, dos registros da sala de operação até a alta ou até o $5^{\circ}$ dia pós-operatório (5० PO), o Que ocorresse primeiro.

De um total inicial de 2657 prontuários foram excluídos 2245 , pois não atendiam aos critérios do estudo. Não foram localizados 1 13, restando 281 prontuários, totalizando 300 cirurgias, visto Que algumas crianças foram submetidas a mais Que uma cirurgia no ano de 2004. Os dados foram armazenados em um banco de dados eletrônico e analisados através de estatística descritiva.

A Tabela I apresenta um panorama geral das cirurgias realizadas em crianças em 2004 nos três hospitais, bem como as cirurgias pesquisadas e as excluídas do estudo.

\section{RESULTADOS E DISCUSSÃO}

Os dados coletados dos 300 prontuários mostram que a maioria das 280 crianças pesquisadas era do sexo masculino $(n=197)$ e 83 do sexo feminino. As idades variaram entre menores de 1 mês de vida até 14 anos incompletos, havendo predominância da faixa etária até 24 meses $(n=59)$. O tempo de internação em 200 cirurgias foi igual ou inferior a 5 dias e a cirurgia pediátrica foi a especialidade com maior número de operações realizadas ( $n=187)$, seguida da ortopedia $(n=55)$, da neurocirurgia $(n=25)$, da cirurgia plástica $(n=10)$, da oftalmologia $(n=9)$ e da otorrinolaringologia $(n=6)$. Em 7 cirurgias pesquisadas foram realizadas intervenções da ortopedia e da cirurgia plástica, e em I caso houve concomitância da cirurgia infantil e da otorrinolaringologia.

Em relação à medicação pré-anestésica, somente antes de 113 das cirurgias pesquisadas ela foi realizada. No entanto, em 264 cirurgias foram administrados analgésicos durante o ato cirúrgico, variando de 1 a 3 tipos de medicamentos por paciente. Em 5 cirurgias, todas no Hospital 1 , em Que as crianças haviam recebido anestesia geral, também foi realizado bloqueio para analgesia pósoperatória, conforme registro dos anestesiologistas. Desses bloqueios, 2 foram do tipo peridural sacral, I peniano, I íleoinguinal e peniano e I caudal.

Um total de 15 crianças recebeu medicação analgésica na SRPA, e, em 194 cirurgias, o início da medicação analgésica na unidade pediátrica ocorreu até duas horas após o retorno do paciente do centro cirúrgico. Apenas em I caso não houve prescrição de analgésicos no pós-operatório imediato, no entanto, em 24 cirurgias não foi administrado analgésico nesse período. Os demais receberam de 1 a 12 doses de analgésicos nas primeiras 24 horas após o término do procedimento cirúrgico, sendo Que o número de doses mais freqüente foi $4(n=85)$. No período pós-operatório imediato (POI) de 76 cirurgias os pacientes receberam de um a três analgésicos no esquema se necessário.

A Tabela 2 apresenta uma caracterização das prescrições médicas com enfoque nos analgésicos e sedativos.

Apesar de que em apenas 37,33\% dos casos pesquisados $(n=112)$ os pacientes receberam medicação pré-anestésica, em $88 \%$ das cirurgias $(n=264)$ foram administrados analgésicos durante o ato cirúrgico indicando uma preocupação dos médicos, mais notadamente dos anestesiologistas, em proporcionar um período pós-operatório de melhor Qualidade em relação à dor. Hoje não se concebe um anestesiologista preocupado apenas com a anestesia cirúrgica e sim vislumbrando o paciente como um todo, o Que inclui os períodos pré e pós-operatório ${ }^{(12)}$. Por outro lado, apesar do fato de Que após todas as cirurgias estudadas os pacientes foram encaminhados à SRPA, apenas em 15 casos foram administrados analgésicos nesse local. Convém frisar Que a dor tratada de maneira preventiva ou o mais precocemente possível é de mais fácil manipulação do Que aQuela já estabelecida ou intensa(1). É importante destacar que o controle da dor aguda pós-operatória é de responsabilidade do anestesista, do cirurgião e da equipe de enfermagem. Esta última deve, na sala de recuperação pósanestésica e na unidade de internação, avaliar a intensidade da dor, aplicar fármacos e técnicas de analgesia e realizar intervenções não-farmacológicas, além de propiciar conforto ao paciente ${ }^{(15)}$.

No $1^{\circ}$ pós-operatório em apenas 8 casos não foi prescrita medicação analgésica. Em 222 cirurgias foi prescrito ao menos um analgésico de horário, mas apenas 210 crianças receberam tal medicação por erro no aprazamento em 12 casos. Em 103 casos, os pacientes receberam dois analgésicos prescritos em horários regulares. $\mathrm{O}$ total de doses recebidas variou de 0 a 12, com maior predominância do número 4 ( $n=53)$.

Ao analisarmos esses dados constatamos Que no $1^{\circ} \mathrm{PO}$ de 79,5\% dos procedimentos cirúrgicos pesquisados foram administrados analgésicos, o Que está de acordo com a recomendação de Que a dor deve ser tratada antes de sua manifestação, isto é, a instituição de um esquema analgésico de horário e não de demanda (se necessário) ${ }^{(12)}$, tendo em vista Que os efeitos deletérios da dor dificultam a recuperação pós-operatória do paciente. É importante lembrar Que a não administração de medicamentos para o controle da dor pós-operatória em doses

Tabela 1. Distribuição de cirurgias realizadas e pes@uisadas nos 3 hospitais.

\begin{tabular}{lcccc}
\hline Dados & Hospital 1 & Hospital 2 & Hospital 3 & Total \\
\hline $\begin{array}{l}\text { Cirurgias e/ou procedimentos realizados } \\
\text { em crianças no centro cirúrgico sob }\end{array}$ & & & & \\
anestesia, no ano de 2004 & 769 & 308 & 1580 & 2657 \\
Prontuários não encontrados & - & - & 113 & 113 \\
Cirurgias/procedimentos excluídos & 624 & 279 & 1341 & 2244 \\
Crianças pesquisadas & 132 & 28 & 120 & 280 \\
Cirurgias pesquisadas & 147 & 29 & 124 & 300 \\
\hline
\end{tabular}


Tabela 2. Caracterização da prescrição e administração de analgésicos e sedativos durante e após as cirurgias nos Hospitais 1,2 e 3 .

\begin{tabular}{|c|c|c|c|c|c|c|c|c|}
\hline \multirow[b]{2}{*}{ Prescrição/Administração } & \multicolumn{8}{|c|}{ Unidade*/Período } \\
\hline & $\begin{array}{c}\text { SO } \\
\mathrm{n}=300\end{array}$ & $\begin{array}{c}\text { SRPA } \\
\mathrm{n}=300\end{array}$ & $\begin{array}{c}\text { POI } \\
\mathrm{n}=300\end{array}$ & $\begin{array}{c}1^{\circ} \mathrm{PO} \\
\mathrm{n}=279\end{array}$ & $\begin{array}{c}2^{\circ} \mathrm{PO} \\
\mathrm{n}=174\end{array}$ & $\begin{array}{c}3^{\circ} \mathrm{PO} \\
\mathrm{n}=116\end{array}$ & $\begin{array}{c}4^{\circ} \mathrm{PO} \\
\mathrm{n}=84\end{array}$ & $\begin{array}{l}5^{\circ} \mathrm{PO} \\
\mathrm{n}=69\end{array}$ \\
\hline $\begin{array}{l}\text { Paciente com prescrição de } \\
\text { analgésico em horário } \\
\text { regular* }\end{array}$ & 264 & 15 & 267 & 222 & 117 & 81 & 61 & 44 \\
\hline $\begin{array}{l}\text { Pacientes Que receberam } \\
\text { analgésicos em horário } \\
\text { regular }\end{array}$ & 264 & 15 & 267 & 210 & 110 & 74 & 59 & 42 \\
\hline $\begin{array}{l}\text { Pacientes com prescrição } \\
\text { de analgésico se necessário } \\
\text { Pacientes Que receberam }\end{array}$ & - & - & 146 & 160 & 118 & 81 & 60 & 58 \\
\hline analgésicos se necessário & - & - & 76 & 59 & 44 & 21 & 18 & 17 \\
\hline $\begin{array}{l}\text { Pacientes sem prescrição } \\
\text { de analgésico }\end{array}$ & 36 & 285 & 1 & 8 & 7 & 8 & 1 & 1 \\
\hline $\begin{array}{l}\text { Pacientes Que não } \\
\text { receberam analgésico }\end{array}$ & 36 & 285 & 25 & 46 & 50 & 31 & 17 & 16 \\
\hline
\end{tabular}

*Unidade/Período : SO = Sala de operação; SRPA = Sala de recuperação pós-anestésica; do POI (pós-operatório imediato) ao 50 PO (pós-operatório) refere-se às Unidades de Internação Pediátrica. **Os analgésicos administrados na SO e SRPA serão in cluídos nessa categoria porém, em sua maioria, foram administrados uma única vez

adequadas nos intervalos planejados, levando o paciente a sofrer dor desnecessária, caracteriza grave desrespeito aos deveres éticos e legais de aliviar o sofrimento causado pela dor ${ }^{(15)}$, conforme o artigo $7^{\circ}$ da Resolução $n^{\circ} 41$ de 13/10/95 - Direitos da Criança e do Adolescente Hospitalizados - do Conselho Nacional dos Direitos da Criança e do Adolescente ${ }^{(16)}$.

No $2^{\circ}$ pós-operatório, em 117 de um total de 174 cirurgias, foram prescritos analgésicos em intervalos regulares; destas, somente 110 pacientes foram medicados devido a erro no aprazamento. Em 118 cirurgias em Que se prescrevia analgésico, apenas se necessário, foram medicadas 44 crianças. Em 7 casos não houve a prescrição de analgésicos, totalizando 50 casos em Que não foi administrado esse tipo de medicamento. As doses administradas variaram de 0 a $1 \mathrm{I}$, novamente com predominância de 4 doses $(n=27)$.

Esses achados indicam Que, dos pacientes Que permaneceram hospitalizados no $2^{\circ} \mathrm{PO}, 66,21 \%(n=117)$ receberam esquema analgésico de horário, um percentual mais baixo Que no $1^{\circ} \mathrm{PO}$. Em $28,57 \%$ das cirurgias $(n=50)$ nenhum analgésico foi administrado nesse período apesar de Que em apenas $4 \%$ dos casos $(n=7)$ esse tipo de medicamento não foi prescrito. Vale lembrar Que não se deve permitir Que o paciente tente tolerar a dor, aguardando que a mesma diminua sozinha, mas sim, ao menor sinal de sua chegada, medicar ou orientar o paciente a receber a medicação analgésica prescrita. Agindo desse modo estaremos contribuindo para impedir a hiperexcitação dos neurônios do corno posterior da medula espinhal (CPME) e Que o fenômeno de wind-up (facilitação para a transmissão de novos impulsos dolorosos vindos da periferia, amplificando a dor do paciente. É considerada uma repercussão da alteração da plasticidade neuronal do sistema nervoso central causada pelo controle inadequado do estímulo doloroso) se estabeleça, com conseQüente amplificação da dor e tornando difícil o seu controle ${ }^{(12)}$.
Os dados obtidos no $3^{\circ} \mathrm{PO}$ indicam que em 81 casos, de 116 cirurgias analisadas foram prescritos analgésicos de horário, mas Que em 7 deles as crianças não receberam o medicamento por erro na colocação do horário, considerando-o como se necessário (em dois casos havia a prescrição de 2 analgésicos de horário e ambos foram considerados de demanda; em outros dois prontuários estavam prescritos 2 analgésicos de horário e um deles foi considerado de demanda). Também em 81 prontuários foram prescritos analgésicos se necessário; em 27 destes se prescrevia analgésico em horário regular. Em 21 casos o analgésico de demanda foi aplicado e em 10 casos essa foi a única forma de administração de analgésicos realizada. O número de doses administradas variou de 0 a 11 , com maior freeüência de doses igual a $4(n=21)$. Esses achados mostram Que em $72,41 \%$ dos casos $(n=84)$ as crianças receberam analgésicos no $3^{\circ} \mathrm{PO}$, Quer seja em horários pré-fixados, Quer seja se necessário ou em ambas as modalidades, percentual mais elevado Que o encontrado no $2^{\circ} \mathrm{PO}$.

Em relação ao $4^{\circ}$ dia de pós-operatório, 61 dos prontuários consultados continham ao menos um analgésico prescrito em horários regulares, variando de 0 a 15 o número de doses administradas. Ocorreu, porém, Que em dois casos as crianças não receberam a medicação aparentemente por descuido do responsável pelo aprazamento das doses, o qual considerou que deveriam ser administradas apenas se necessário. Em 60 casos foram prescritos analgésicos se necessário no $4^{\circ} \mathrm{PO}$ ( 18 crianças foram medicadas dessa maneira); desse total, 38 receberam esse tipo de medicamento em horário regular. Apenas em um caso não foi prescrito analgésico no $4^{\circ} \mathrm{PO}$.

Em razão dos dados coletados notamos que em 80\% dos procedimentos cirúrgicos pesquisados Que obrigaram as crianças a permanecer internadas no $4^{\circ} \mathrm{PO}$, estas receberam analgésicos em horários regulares ou, caso houvesse, no esQuema de demanda. Somente em um caso não houve prescrição de analgésicos. Em 
45,23\% dos casos foram prescritos analgésicos em horários regulares e também se necessário.

Em 44 prontuários das crianças Que permaneceram internadas até o $5^{\circ} \mathrm{PO}(\mathrm{n}=69)$ havia sido prescrito no mínimo um analgésico a ser administrado em horários regulares, sendo Que as doses administradas variaram de 0 a 14 , sendo 4 o número de doses mais freeüente. Por erro no aprazamento em dois prontuários (a medicação ficou como se necessário), apenas 42 crianças receberam esse tipo de medicação em intervalos predeterminados. Em 58 prontuários encontramos analgésicos prescritos na modalidade se necessário, mas apenas 17 crianças receberam esse tipo de medicação no $5^{\circ} \mathrm{PO}$, o Que aponta para um total de 53 casos em Que houve administração de ao menos um analgésico independente do esQuema proposto. Os dados indicam Que $76,81 \%$ das crianças Que permaneceram hospitalizadas até o $5^{\circ} \mathrm{PO}$ receberam ao menos uma dose de analgésico, número muito próximo ao obtido no $4^{\circ} \mathrm{PO}$.

Relembramos que o controle inadequado da dor pós-operatória pode provocar alterações fisiológicas, bioquímicas e estruturais no sistema nervoso central ${ }^{(12)}$, daí a necessidade premente de Que seu alívio seja efetuado no menor tempo possível.

Os resultados apresentados nos levam a refletir sobre o preparo dos profissionais envolvidos na assistência à criança no período pós-operatório e sobre o processo de trabalho adotado nas unidades pesquisadas relacionado à administração de medicamentos analgésicos. Nos locais estudados o profissional responsável pelo aprazamento era o auxiliar técnico-administrativo. Esta tarefa, delegada a este profissional pela enfermeira do setor, é de extrema importância e Quando realizada de forma inadequada pode trazer conseqüências indesejadas, no Que se refere ao controle da dor pós-operatória pediátrica. Em alguns casos o aprazamento indevido perdurou por mais de um dia, não sendo percebido nem pelos médicos nem pelos integrantes da equipe de enfermagem.

Convém destacar Que a falta de preparo e de atualização técnica e científica, a falta de vontade ou de condições adequadas de trabalho do profissional de saúde em assistir de modo efetivo ao paciente com dor pós-operatória, pode ser considerada um séria Quebra de sua responsabilidade ética e grave lesão aos preceitos da Declaração Universal dos Direitos Humanos ${ }^{(15)}$.

Outro importante aspecto a ser destacado é Que o profissional de saúde não pode garantir a ausência completa da dor após a cirurgia, mas é sim possível e necessário aliviar essa dor, tornandoa pelo menos suportável, facilitando a recuperação do paciente e evitando deixar lembranças desagradáveis ${ }^{(15)}$.

Analisando de uma forma mais ampla os dados extraídos dos prontuários em relação aos registros da dor pós-operatória e seu manejo na SRPA e unidade pediátrica, incluindo o uso de medicação, comunicação da dor ao médico, reavaliação da criança e estratégias não-farmacológicas, observamos a discrepância entre o número de registros efetuados por enfermeiras e os efetuados por auxiliares ou técnicos de enfermagem. Se considerarmos cada hospital separadamente, encontramos os seguintes resultados de registros feitos por enfermeiras desde a SRPA até o $5^{\circ}$ PO: Hospital I, 10 registros; Hospital 2 com 8 registros e Hospital 3 com 182 registros. Atentando para a grande diferença numérica de cirurgias pesQuisadas nos Hospitais 1 e 2, 147 e 29 cirurgias, respectivamente, vemos Que esse número aponta para a falta de sistematização das enfermeiras do Hospital 1 no registro de informações sobre avaliação e manejo da dor pós-operatória nos prontuários consultados.

Ao compararmos o número de cirurgias pesquisadas nos Hospitais I e 3, 147 e 124, respectivamente, encontramos números próximos, porém, o número de registros de enfermeiras apresenta grande variação, a saber, 182 registros no Hospital 3 e os 10 já mencionados no Hospital 1 . Se considerarmos a relação leito/enfermeira o índice do Hospital I é de 5,66 leitos por enfermeira; o do Hospital 2 de 4 leitos por enfermeira e o do Hospital 3 de 10,5 leitos por enfermeira. Portanto, embora a relação leito/enfermeira seja a mais desfavorável dentre os hospitais pesquisados o Hospital 3 apresentou o maior número de registros feitos por enfermeiras. Vale frisar que os 3 hospitais pesquisados recebem alunos do curso de graduação em Enfermagem, mas os prontuários pesquisados e Que traziam registros dos alunos sobre dor pós-operatória foram excluídos.

Quanto ao total de registros realizados por auxiliares ou técnicos de enfermagem, a situação se inverte. Tomando como exemplo o item comentários da criança revelando dor com 247 registros, constatamos Que $195(78,94 \%)$ desse total foram obtidos do Hospital 1 .

É preciso também levar em conta a grande diferença encontrada Quanto ao número de funcionários dessas duas unidades pediátricas. No Hospital I há 35 auxiliares e técnicos de enfermagem Que se revezam nas 24 horas para atender 34 leitos pediátricos. Já o Hospital 3 possui 24 auxiliares e técnicos de enfermagem e 42 leitos na unidade pediátrica. Se considerarmos a relação entre funcionário e leito temos no Hospital I a proporção de 0,97 leito por funcionário e no Hospital 3 a proporção de 1,75 leito por funcionário. No Hospital 2 essa proporção é de 1,42 funcionário por leito. Portanto, o reduzido número de funcionários do Hospital 3 pode explicar parcialmente o pequeno número de registros efetuados por auxiliares e técnicos Quando comparados com os registros feitos nos demais hospitais. Outro ponto a ser ressaltado é o fato dos Hospitais 2 e 3 serem privados e o Hospital I ser público, o que também pode contribuir para Que os primeiros tenham um Quadro de pessoal mais enxuto, visando o controle de gastos.

O item com maior número de registros, 393, foi o relacionado à administração de analgésicos, conforme prescrição médica com 358 citações de auxiliares ou técnicos de enfermagem. Novamente o maior número desses registros ficou concentrado no Hospital I, responsável por $304(84,91 \%)$ do total dos dados. No entanto, esse mesmo item só foi registrado pelas enfermeiras do Hospital 2.

Considerando os registros sobre o uso de estratégias nãofarmacológicas (uso de arco protetor, companhia/colo/presença dos pais, elevação de partes do corpo), observamos Que os registros foram feitos unicamente por auxiliares ou técnicos de enfermagem.

A Tabela 3 apresenta dados sobre os itens prescritos pelas enfermeiras referentes à dor pós-operatória.

Em todo o período pesquisado Que compreendeu do POI ao $5^{\circ}$ PO a porcentagem de prescrições de enfermagem Que possuía algum item relacionado à dor pós-operatória variou de $66,33 \%$ $(n=199)$ no POI, a 53,62\% ( $n=37)$ no $5^{\circ}$ PO.

Apesar da prescrição solicitar a anotação de sinais e Queixas de dor, esses registros são pouco anotados mesmo quando a criança é submetida a procedimentos potencialmente dolorosos. Vale ressaltar Que em Quatro casos cujas prescrições de enfermagem continham o item observar e anotar Queixas de dor, as crianças 
Tabela 3. Itens na prescrição de enfermagem referentes à dor pós-operatória dos Hospitais 1, 2 e 3.

\begin{tabular}{|c|c|c|c|c|c|c|c|}
\hline \multirow[b]{2}{*}{ Itens } & \multicolumn{7}{|c|}{ Dia de Pós-operatório } \\
\hline & $\begin{array}{c}\text { POI } \\
(n=300)\end{array}$ & $\begin{array}{c}10 \mathrm{PO} \\
(\mathrm{n}=279) \\
\end{array}$ & $\begin{array}{c}2^{\circ} \mathrm{PO} \\
(\mathrm{n}=172)\end{array}$ & $\begin{array}{l}3^{\circ} \mathrm{PO} \\
(\mathrm{n}=115)\end{array}$ & $\begin{array}{l}4^{\circ} \mathrm{PO} \\
(\mathrm{n}=83)\end{array}$ & $\begin{array}{l}5^{\circ} \mathrm{PO} \\
(\mathrm{n}=69)\end{array}$ & Total \\
\hline $\begin{array}{l}\text { Com item relacionado à dor pós- } \\
\text { operatória }\end{array}$ & 199 & 183 & 103 & 72 & 51 & 37 & \# \\
\hline $\begin{array}{l}\text { Observar e anotar sinais/fácies de dor } \\
\text { Observar e anotar dor em }\end{array}$ & 2 & 5 & 4 & 4 & 3 & 1 & 19 \\
\hline local/parte/membro operado & 15 & 7 & 6 & 5 & 2 & 3 & 38 \\
\hline Observar e anotar Queixas de dor & 66 & 78 & 38 & 23 & 22 & 14 & 241 \\
\hline Observar e anotar dor(es) & 109 & 87 & 50 & 34 & 21 & 21 & 322 \\
\hline Observar e anotar algia(s) & 18 & 12 & 8 & 7 & 4 & 4 & 53 \\
\hline $\begin{array}{l}\text { Outros itens } \\
\text { Prescrição de estratégia não- } \\
\text { farmacológica para alívio da dor }\end{array}$ & 2 & 3 & 3 & 3 & 3 & 1 & 15 \\
\hline $\begin{array}{l}\text { Manter arco protetor } \\
\text { Elevar parte do corpo/membro }\end{array}$ & 6 & 7 & 6 & 6 & 6 & 6 & 37 \\
\hline operado & 17 & 21 & 6 & 4 & 1 & 1 & 50 \\
\hline Outras & 1 & 1 & 1 & - & 1 & 2 & 6 \\
\hline
\end{tabular}

eram menores de um ano, portanto sem capacidade de verbalização da dor. Esses achados são semelhantes aos de um estudo Que analisou registros de enfermagem sobre dor e analgesia, e indicaram Que, em geral, a prescrição de enfermagem foi vaga Quanto ao controle álgico ${ }^{(17)}$.

Como apenas $11,9 \%(n=93)$ dos itens relacionavam-se à prescrição de estratégias não-farmacológicas para o alívio da dor, fica evidente a pouca utilização deste tipo de método no controle da dor pediátrica nos 3 hospitais pesquisados. A administração de medicamentos é a base para o alívio efetivo da dor, mas em virtude da dor ser mais Que meramente uma experiência sensorial, os métodos não-farmacológicos para seu alívio são parte importante do cuidado ao paciente pediátrico ${ }^{(18)}$.

\section{CONCLUSÃO}

O alívio da dor humana é preceito defendido há milênios e considerado importante missão dos profissionais da área da saúde. Mesmo com o significativo aumento de estudos relacionados à dor, inclusive aquela Que acomete a criança, o conhecimento gerado tem sido pouco aplicado na prática de enfermagem.

A pesquisa nos prontuários das crianças submetidas à cirurgia contribuiu de maneira decisiva no delineamento do panorama da assistência prestada aos pacientes com dor no período pósoperatório na sala de cirurgia, na SRPA e na unidade pediátrica. Os dados obtidos denotam a pouca valorização pelas enfermeiras em registrar os sinais e/ou Queixas de dor dos pacientes, bem como as medidas adotadas para avaliação e controle da dor e os resultados das intervenções realizadas. Em cerca de metade das prescrições de enfermagem havia ao menos um item relacionado à dor pósoperatória, o Que indica um cenário mais animador. No entanto, alguns itens eram bastante vagos e, por vezes, não se aplicavam à faixa etária da criança.

Encontramos prontuários com erros no aprazamento dos analgésicos Que, em alguns casos, persistiram por vários dias, indicando despreparo dos funcionários técnico-administrativos Que executam essa atividade e recebem supervisão inadequada. Porém, mais grave Que a ocorrência dessas falhas em si mesmas, é a constatação de Que várias crianças deixaram de receber analgésicos num período potencialmente doloroso como o período pósoperatório e, conseQüentemente, sentiram dor.

O manejo adequado da dor tem sido pouco valorizado e realizado em nosso sistema de saúde. Os prontuários pesquisados revelaram razoável cobertura analgésica medicamentosa nas prescrições médicas, ao mesmo tempo em Que evidenciam a inexistência de avaliação sistematizada da dor pós-operatória de QualQuer natureza, bem como ausência ou escassez de registros de enfermagem relacionados à dor pós-operatória e/ou aos efeitos do tratamento adotado para seu alívio. Esses achados evidenciam a necessidade de realização de pesquisas sobre manejo da dor pediátrica, os fatores contextuais Que o influenciam e as atitudes e valores das enfermeiras em relação ao manejo da dor das crianças.

\section{REFERÊNCIAS}

1. Howard RF. Current status of pain management in children. IAMA 2003; 290( I 8): 2464-69.

2. Helgadóttir HL. Pain management practices in children after surgery. I Pediatric Nurs 2000; $15(5)$ : 334-40.

3. Salanterä S, Lauri S, Salmi TT, Aantaa R. Nursing activities and autocomes of care in the assessment, management, and documentation of children's pain. I Pediatric Nurs 1999;
14(6): 408-15.

4. American Academy of Pediatrics. American Pain Society. The assessment and management of acute pain in infants, children, and adolescents. Pediatrics 2001; I08(3): 793-7.

5. Carvalho M. Dor no recém-nascido. Pediatria Moderna 1995; 3 1 (6): 925-34.

6. Carvalho WB. Analgesia e sedação em pediatria. Rev Paul 
Registros sobre dor pós-operatória em crianças: uma análise retrospectiva de hospitais de Londrina, PR, Brasil

Pediatria 1996; 14(2): 53-65.

7. Rossato LM, Rezende MA. A criança com dor. In: Sigaud CHS, Veríssimo MDLÓ, organizadoras. Enfermagem pediátrica: o cuidado de enfermagem à criança e ao adolescente. São Paulo (SP): EPU; 1996. p. 223-9.

8. Coeli SCM. Dor aguda na criança. In: Drummond JP. Dor aguda: fisiopatologia, clínica e terapêutica. São Paulo (SP): Atheneu; 2000. p. 151-70.

9. Carvalho WB, Troster JE. Sedação e analgesia em prontosocorro. I Pediatria 1999; 75(Supl 2): 294-306.

10. Barbosa SMM, Guinsburg R. Dor de acordo com as faixas etárias pediátricas. In: Teixeira MJ, editor. Dor: contexto interdisciplinar. Curitiba (PR): Editora Maio; 2003. p. 536-45.

I1. Cavalcante VO, Teixeira MJ, Franco RA. Dor pós-operatória. Rev Simbidor 2000; I (1): 45-53.

12. Andrade MP. Dor pós-operatória: conceitos básicos de fisiopatologia e tratamento. Rev Soc Bras Estudos Dor 2000; 2(2): 7-14.

13. Garcia JBS, Sakata RK, Issy A M. Analgesia preemptiva. In: Teixeira MI, editor. Dor: contexto interdisciplinar. Curitiba
(PR): Editora Maio; 2003. p. 755-62.

14. Silva YB, Pimenta CAM. Avaliação e registro inadequados da Queixa de dor: barreira a ser superada. In: Arquivos do $6^{\circ}$ Simpósio Brasileiro e Encontro Internacional sobre Dor; 2003 maio 8-10; São Paulo (SP), Brasil. São Paulo (SP): Lemos Editorial \& Gráficos; 2003. p. 13-8.

15. Posso IP, Costa, DSP. De Quem é a responsabilidade no tratamento da dor pós-operatória? Rev Âmbito Hospitalar 2005; 17(170): 3-8.

16. Ministério da Justiça (BR). Conselho Nacional dos Direitos da Criança e do Adolescente. Direitos da Criança e do Adolescente Hospitalizados: Resolução no 4l/95. Diário Oficial, Brasília (DF), 17 out. 1995; 16319-20;

17. Silva YB, Pimenta CAM. Análise dos registros de enfermagem sobre dor e analgesia em doentes hospitalizados. Rev Esc Enferm USP 2003; 37(2): 109-18.

18. Pölkki T, Laukkala H,. Vehviläinen-Julkunen K, Pietilä A. Factors influencing nurses' use of nonpharmacological pain alleviation methods in paediatric patients. Scand I Caring Sci 2003; 17(4): 373-83. 